NASA Technical Memorandum 100858 ICOMP-88-9

\title{
Evolution of Hairpin Vortices in a Shear Flow
}

T.-L. Hon

Lehigh University

Bethlehem, Pennsylvania

and

J.D.A. Walker

Institute for Computational Mechanics in Propulsion Lewis Research Center Cleveland, Ohio

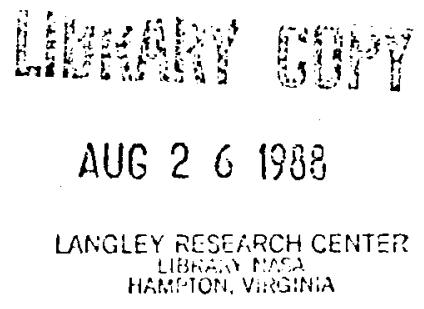

July 1988

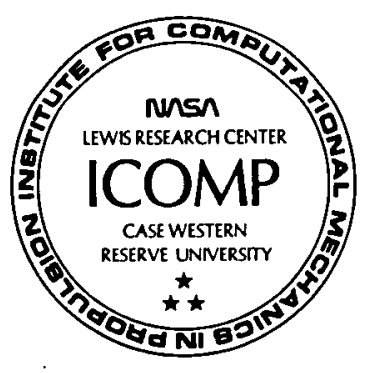




\title{
EVOLUTION OF HAIRPIN VORTICES IN A SHEAR FLOW
}

\author{
T. - L. Hon \\ Department of Mechanical Engineering and Mechanics \\ Lehigh University \\ Bethlehem, Pennsylvania 18015 \\ and \\ J.D.A. Walker* \\ Institute for Computational Mechanics in Propulsion \\ National Aeronautics and Space Administration \\ Lewis Research Center \\ Cleveland, Ohio 44135
}

SUMMARY

Recent experimental studies suggest that the hairpin vortex plays an important (and perhaps dominant) role in the dynamics of turbulent flows near walls. In this study a numerical procedure is developed to allow the accurate computation of the trajectory of a three-dimensional vortex having a small core radius. For hairpin vortices which are convected in a shear flow above a wall, the calculated results show that a two-dimensional vortex containing a small three-dimensional disturbance distorts into a complex shape with subsidiary hairpin vortices forming outboard of the original hairpin vortex. As the vortex moves above the wall, it induces unsteady motion in the viscous flow near the wall; numerical solutions suggest that the boundary-layer flow near the wall will ultimately erupt in response to the motion of the hairpin vortex and in the process a secondary hairpin vortex will be created. The computed results agree with recent experimental observations.

\section{INTRODUCTION}

It has often been suggested that the hairpin vortex is a basic building block in the dynamics of turbulent flows near walls (refs. 1 to 5 ). Such vortices have been observed in a number of careful experimental studies (refs. 2, 4, and 5). The experiments of Acarlar and Smith (refs. 4 and 5) were carried out by introducing hairpin vortices into an otherwise laminar boundary layer and subsequently observing their evolution as well as their effects on the rest of the flow; these experiments suggest that hairpin vortices are regenerative in at least two ways: (1) a hairpin vortex convected in a shear flow appears to be able to multiply itself in the spanwise direction in the sense that subsidiary hairpin vortices were observed to form outboard of the original hairpin vortex and (2) hairpin vortices appear to actuate the creation of secondary hairpin vortices through an eruption of the viscous flow near walls and a subsequent viscous-inviscid interaction. In this computational study, phenomena directly relevant to the

*Work funded under Space Act Agreement C99066G; presently at Department of Mechanical Engineering and Mechanics, Lehigh University, Bethlehem, Pennsylvania 18015. 
experiments of Acarlar and Smith (refs. 4 and 5) will be addressed. In particular, calculations related to two aspects were carried out: (1) the time-dependent evolution of a hairpin vortex in a shear flow above a wall and (2) the response of the viscous flow near the wall as the hairpin vortex passes by. Since the Reynolds numbers associated with the vortex motions within turbulent boundary layers are large, the subsequent analys is is carried out based on equations and concepts which are valid in the limit of infinite Reynolds number; here the objective is to determine basic fluid mechanics phenomena and trends that are realized asymptotically over a range of Reynolds numbers. In such circumstances, there are two (and eventually three) regions of the flow field where the effects of viscosity are important. The hairpin vortex is taken to convect in an effectively inviscid flow above the wall but viscosity is important near the vortex core where sharp variations in the flow field occur locally; viscosity is also important near the wall where the moving vortex induces an unsteady boundary-layer effect. Eventually moving vortices are known to trigger an unsteady separation effect near the wall (refs. 6 and 7) and to cause an eruption of the flow near the wall; in such circumstances, moving viscous layers erupt from the wall and penetrate into the outer inviscid region in an interaction which is complex (refs. 8 and 9) and (as yet) not well understood. In the present study, only events leading up to this last type of phenomenon will be considered.

It is worthwhile to mention that in recent times, there has been an increasing interest in performing direct numerical simulations of turbulent flows such as fully-developed turbulent flows in channels (refs. 10 and 11). In such computations, the full three-dimensional unsteady Navier-Stokes equations are solved over a period of time for a limited portion of the channel, subject to various assumptions concerning periodicity in the spatial directions. One objective of such studies is to try to develop an appreciation of the complex physical processes that take place in the turbulence, particularly near solid walls. At present, it has only been possible to perform these simulations for fairly low Reynolds numbers and with numerical grids that give rather limited spatial resolution. Inevitably, large masses of numerical data are generated in this approach and to attempt to answer questions concerning cause and effect relationships, it is necessary to search through the data records for events which appear significant and to then make some type of interpretation. Despite the aforementioned difficulties, it has been found (ref. 11) (by tracing estimates of the instantaneous vortex lines) that there is evidence of vortex structures in the computed results which appear to be similar to hairpin vortices.

In the present study, a somewhat more fundamental approach was taken, which was guided to a large extent by experimental observations. The studies of Head and Bandyophadyay (ref. 2) and Acarlar and Smith (refs. 4 and 5) provide convincing evidence that hairpin vortices are an important dynamical feature of turbulent flows near walls. Consequently the objective of this study was to develop a better understanding of the dynamics of a convected hairpin vortex near a wall, as well as the type of viscous flow response close to the wall. In the near-wall region, a turbulent flow is highly sheared in the streamwise direction; therefore the evolution of a hairpin vortex in a linear shear flow near a wall was considered to be the simplest well-defined problem which nevertheless captures many of the features of the environment of a turbulent flow near a wall. The general problem of hairpin vortex evolution is complicated since it is necessary to numerically compute the trajectory of a three-dimensional space curve, which carries a viscous core region with it 
and which deforms with time into complex shapes. In the present study, the hairpin vortex was assumed to initiate as a small perturbation in an otherwise two-dimensional vortex.

\section{VORTEX CALCULATION METHODS}

In situations where the vorticity is concentrated in vortex tubes of small cross-section, the evolution of the flow field is readily computed using Lagrangian methods. For a single vortex tube with circulation $\Gamma$, a vortex Reynolds number may be defined as $\Gamma / v$, where $v$ is the kinematic viscosity; for large vortex Reynolds numbers, the cross-sectional area of the vortex core region is generally small and at any instant the vortex position is defined as a three-dimensional space curve. The instantaneous flow due to the vortex is described by the Biot-Savart law, viz.:

$$
\vec{u}\left(\vec{x}_{0}\right)=-\frac{\Gamma}{4 \pi} \int_{C} \frac{\left(\vec{x}_{0}-\vec{x}\right)}{\left|\vec{x}_{0}-\vec{x}\right|^{3}} \times d \vec{x}+\vec{u}_{e x t}\left(\vec{x}_{0}\right) \text {. }
$$

Here $(x, y, z)$ are Cartesian coordinates as indicated in figure $1, \vec{x}_{0}$ is a position vector to an arbitrary location in space and $\vec{u}$ ext is a background velocity field; $s$ measures distance along the curve $c$ defining the vortex and $\hat{t}$ is the unit tangent. The sense of positive vorticity is given by the right-hand rule and for the situation depicted in figure 1 , the vortex has negative rotation. Note that according to Kelvin's theorem, the circulation $\Gamma$ about the vortex core is constant in time and along the length of the vortex.

Since $\vec{u}\left(\vec{x}_{0}\right)=\partial \vec{x}_{0} / \partial t$, equation (1) apparently defines a differentia, equation to track the trajectory of each point on the vortex forward in time. However as $\vec{X}_{0}$ approaches a location on the curve $C$, the integrand in equation (1) becomes singular and consequently the velocity field described by the Biot-Savart law must be interpreted as an outer inviscid solution which is not uniformly valid near the vortex core. To define a rational calculation procedure, it is necessary to match any solution obtained from equation ( 1 ) with an inner viscous solution in the vortex core and thereby take into account how the evolving core flow influences the overall vortex motion. A general method of this nature has been described by Callegari and Ting (ref. 12) for vortices which form closed loops. At present there is no corresponding theoretical framework for infinitely long vortices. However an alternative approach which has proved effective for closed loop vortices and which gives essentially similar results is due to Moore (ref. 13); in this method the singularity in equation (1) is avoided by artificially introducing a small parameter $\mu$ in the denominator of the integrand. Generally $\mu$ is proportional to the core radius a and the precise relationship is established by insisting that the integration formula collapse to give known exact results for a translating circular vortex ring in an infinite and otherwise stagnant medium. In this manner, $\mu / a$ is obtained as an expression involving integrals of the swirl and axial velocities in the vortex core (refs. 14 and 15). A further feature of Moore's (ref. 13) algorithm was motivated by considerations of numerical accuracy; for small values of a and hence $\mu$, the integrand varies rapidiy near $X=X_{0}$ and Moore (ref. 13) 
obtained the leading term in an expansion about this point. If $s$ denotes a Lagrangian coordinate measured along the vortex and $s_{0}$ is a specific point, the form of Moore's algorithm is

$$
\begin{array}{r}
\vec{u}\left(s_{0}, t\right)=\frac{\Gamma}{4 \pi} \int_{C}\left[\frac{\partial \vec{x}}{\partial s} \times \frac{\left(\vec{x}_{0}-\vec{x}\right)}{\left(\left|\vec{x}_{0}-\vec{x}\right|^{2}+\mu^{2}\right)^{3 / 2}}-\left(\frac{\partial \vec{x}}{\partial s}\right)_{0} \times\left(\frac{\partial^{2} \vec{x}}{\partial s^{2}}\right)_{0} P(s)\right] d s \\
+\frac{\Gamma}{4 \pi}\left(\frac{\partial \vec{x}}{\partial s}\right)_{0} \times\left(\frac{\partial^{2} \vec{x}}{\partial s^{2}}\right)_{0} P(s) d s+\vec{u}_{C},
\end{array}
$$

where

$$
P(s)=\frac{0.5\left(s-s_{0}\right)^{2}}{\left[\left(s-s_{0}\right)^{2}\left(\frac{\partial \vec{X}}{\partial s}\right)_{0}^{2}+\mu^{2}\right]^{3 / 2}} .
$$

In these equations, the subscript 0 indicates that a quantity is evaluated at $s=s_{0}$. The apparent advantage of this procedure is that the integrand in the first integral in:equation (2) is $0(1)$ everywhere while the second integral is easily evaluated analytically (ref. 16).

This vortex calculation method has been used with good success by various authors (refs. 13, 17, and 18); however in all these studies fairly large values of the vortex core were used $(a \sim 0.1$ to 0.3$)$. In the present study the objective was to compute the evolution of hairpin vortices close to solid walls and to avoid situations where the vortex core impacted the wall at relatively early stages; thus it was considered desirable to be able to calculate cases with very small vortex cores. Unfortunately Moore's (ref. 13) algorithm exhibits strong numerical instability for small values of a (ref. 16); this instability becomes worse when the spatial mesh size along the vortex is reduced and results in the rapid evolution of unrealistic sharp corners in the calculated curves defining the instantaneous vortex position. This problem is associated with the numerical evaluation of the first term in the integrand of equation (2); for small $\mu$, numerical errors in the evaluation of $\partial \vec{X} / \partial s$ are magnified by the small denominator near $s=s_{0}$. If $R\left(s, s_{0}\right)$ denotes the integrand in the first integral in equation (2), it can be shown (ref. 16) that

$$
\vec{R}\left(s, s_{0}\right)=\vec{A}_{1}\left(s-s_{0}\right)^{3}+\vec{A}_{2}\left(s-s_{0}\right)^{4} \text {, }
$$

where $\vec{A}_{1}$ and $\vec{A}_{2}$ are vectors defined by

$$
\vec{A}_{1}=\frac{1}{3 \gamma^{3 / 2}} \vec{X}_{0}^{\prime} \times \vec{X}_{0}^{\prime \prime \prime}-\frac{3}{4 \gamma^{5 / 2}}\left(\vec{X}_{0}^{\prime} \cdot \vec{X}_{0}^{\prime}{ }^{\prime}\right)\left(\vec{X}_{0}^{\prime} \times \vec{X}_{0}\right)\left(s-s_{0}\right)^{2}+\cdots,
$$




$$
\begin{aligned}
& \vec{A}_{2}=\frac{1}{24 \gamma^{3 / 2}}\left(3 \vec{X}_{0}^{\prime} \times \vec{X}_{0}^{\prime}{ }^{\prime \prime}+2 \vec{X}_{0}^{\prime} \times \vec{X}_{0}^{\prime \prime}{ }^{\prime}\right)-\frac{1}{2 \gamma^{5 / 2}}\left(\vec{X}_{0}^{\prime} \cdot \vec{X}_{0}^{\prime}\right)\left(\vec{X}_{0}^{\prime} \times \vec{X}_{0}^{\prime \prime}{ }^{\prime}\right)\left(s-s_{0}\right)^{2} \\
& -\frac{1}{16 \gamma^{5 / 2}}\left(3 \vec{X}_{0}^{\prime} \cdot \cdot \vec{X}_{0}^{\prime \prime}+4 \vec{X}_{0}^{\prime} \cdot \vec{X}_{0}^{\prime \prime \prime}\right)\left(\vec{X}_{0}^{\prime} \times \vec{X}_{0}^{\prime \prime}\right)\left(s-s_{0}\right)^{2}+\ldots \cdot
\end{aligned}
$$

Here the primes denote differentiation along the vortex with respect to $s$, a subscript 0 indicates that a quantity is evaluated as $s=s_{0}$ and $Y$ is defined by

$$
\gamma=\left(s-s_{0}\right)^{2}\left(\vec{X}_{0}^{\prime}\right)^{2}+\mu^{2} .
$$

A calculation procedure may then be formulated in which the first integrand in equation (2) is represented locally near $s=s_{0}$ by the first two terms in the expansion (4) and outside this range by :onventional numerical evaluation. Note that the local interval near $s=s_{0}$, for which the expansion (4) may be applied with good accuracy, is dependent on the mesh used to discretize the vortex as well as the instantaneous shape of the vortex. In practice, the representation (4) was used at the first one or two mesh points on either side of $s=s_{0}$.

\section{THE HAIRPIN VORTEX}

The hairpin vortex was assumed here to initiate as a small threedimensional disturbance in an otherwise two-dimensional vortex. It is convenient to define dimensionless variables and to this end consider the situation depicted in figure $2(a)$ where a rectilinear vortex of strength $k$ is located a distance $d$ above a plane wall. For the indicated sense of circulation, inviscid theory predicts that the vortex will move continuously to the left with self-induced speed

$$
U_{0}=\kappa / 2 d,
$$

and remain at constant height $d$ above the wall. Now consider the more complex situation depicted in figures $2(b)$ and (c) where a hairpin vortex is in motion above the wall. At large distances from the hairpin portion, the vortex will continue to behave as if two-dimensional. It is therefore convenient to define dimensionless variables in terms of $d$ and the self-induced speed in equation ( 8 ); all following equations are assumed to be in terms of these dimensionless variables. Note that the magnitude of the circulation is related to the vortex strength $\kappa$ by $|\Gamma|=2 \pi \kappa$ and for the situations of interest here $\Gamma<0$.

The initial vortex configuration used is of the form

$$
\vec{X}(s, t)=A[(\cos \alpha) \hat{i}+(\sin \alpha) \hat{j}] e^{-\beta s^{2}}+\hat{j}+s \hat{k} .
$$

Here $(\hat{i}, \hat{j}, \hat{k})$ are unit vectors in the $(x, y, z)$ directions corresponding to the streamwise, normal and spanwise directions respectively. This configuration is a two-dimensional vortex located at a distance 1 from the wall with a 
three-dimensional distortion which is symmetrical about $s=0$; the parameter $s$ is a Lagrangian coordinate which ranges from $-\infty$ to $\infty$ along the vortex. In addition $A$ represents the amplitude of the distortion and $\alpha$ is the angle that the plane of the distortion makes with the wall; $\beta$ is a (large) number whose value determines the effective spanwise width of the initial distortion.

- For a vortex of infinite length which is convected in a background flow $U_{b} \hat{i}$ above a plane wall, the equation of motion is obtained from (2) and in dimensionless variables is of the form

$$
\frac{\partial \vec{X}}{\partial t}\left(s_{0}, t\right)=\sum_{j=1}^{7} \vec{I}_{j}\left(s_{0}, t\right)+\vec{u}_{e x t} .
$$

Here so denotes a typical nodal point on the vortex and the vector integrals $\vec{I}_{j}$ will help be defined in the following discussion. The integral in equation (2) along the vortex is split into three parts corresponding to: a symmetric interval ( $\left.s_{0}-h, s_{0}+h\right)$ where the representation (4) of the integrand is used; (2) integrals over the main curved part of the vortex and (3) the range outboard of the disturbance where $|s|>\&$ and where the vortex was assumed to remain a straight line. The half-length of the interval about so must be small enough so that equation (4) gives a convergent representation of the integrand; after some trials, it was determined (for the mesh distributions used in this study) that a convenient choice for $h$ corresponded to one mesh length in $s$. For $|s|>\ell$, the equation of the vortex is

$$
\vec{x}(s, t)=\left(U_{b}-t\right) \hat{i}+\hat{j}+s \hat{k}, \quad|s|>\ell .
$$

It should be noted that $\ell$ must be selected large enough to ensure that there is no significant effect of $l$ on the development of the hairpin vortex; as the vortex disturbance spread with time in the spanwise direction, it was necessary to continually increase the value of $l$.

The first vector integrals in equation (10) correspond to integrations along the main curved part of the vortex and

$$
\vec{I}_{1}=\int_{-\ell}^{-h} \vec{Q}_{V}\left(s, s_{0}, t\right) d s, \quad \vec{I}_{2}=\int_{h}^{l} \vec{Q}_{V}\left(s, s_{0}, t\right) d s,
$$

where

$$
\vec{Q}_{v}=-\frac{\partial \vec{X}}{\partial s} \times \frac{(\vec{X} 0-\vec{X})}{\left(\left|\vec{X}_{0}-\vec{X}\right|^{2}+\mu^{2}\right)^{3 / 2}}+\left(\frac{\partial \vec{x}}{\partial s}\right)_{0} \times\left(\frac{\partial^{2} \vec{x}}{\partial s^{2}}\right)_{0} P\left(s, s_{0}\right) .
$$

The vector integral near the field point $s=s_{0}$ is given by 


$$
\vec{I}_{3}=\int_{-h}^{h} \vec{R}\left(s, s_{0}, t\right) d s
$$

where $\vec{R}$ is given by equation (4). The vector integral over the straight portions of the vortex is

$$
\vec{I}_{4}=\int_{-\infty}^{-l} \vec{Q}_{v \infty} d s+\int_{\ell}^{\infty} \vec{Q}_{V \infty} d s
$$

where

$$
\vec{Q}_{v \infty}=-\frac{\partial \vec{X}}{\partial s} \times \frac{\left(\vec{x}_{0}-\vec{x}\right)}{\left(\left|\vec{x}_{0}-\vec{x}\right|^{2}+\mu^{2}\right)^{3 / 2}} .
$$

Here $\vec{x}$ is evaluated along the straight portion of the vortex and is given by equation (11); an analytical expression for $\vec{I}_{4}$ is readily obtained through a straightforward integration (ref. 16). The vector integral $\mathbb{I}_{5}$ is given by

$$
\vec{I}_{5}=\left(\frac{\partial \vec{X}}{\partial s}\right)_{0} \times\left(\frac{\partial^{2} \vec{X}}{\partial s^{2}}\right)_{0} \int_{-\ell}^{\ell} P\left(s, s_{0}\right) d s,
$$

which is also easily evaluated analytically (ref. 16). Finally $\vec{I}_{6}$ and $\vec{I}_{7}$ represent integrals over the curved and straight portions of the image vortex below the wall, respectively, and

$$
\vec{I}_{6}=\int_{-\ell}^{\ell} \vec{Q}_{j} d s, \quad \vec{I}_{7}=\int_{-\ell}^{-\infty} \vec{Q}_{i} d s+\int_{\ell}^{\infty} \vec{Q}_{i} d s .
$$

Here

$$
\vec{Q}_{i}=\frac{\partial \vec{x}_{i}}{\partial s} \times \frac{\left(\vec{x}_{0}-\vec{x}_{i}\right)}{\left|\vec{x}_{0}-\vec{x}_{j}\right|^{3}},
$$

and $\vec{x}_{j}$ denotes a vector to an arbitrary location on the image vortex below the wall; $\vec{I}_{7}$ may also be evaluated analytically (ref. 16).

\section{CALCULATED VORTEX TRAJECTORIES}

Starting from the initial vortex configuration in equation (9), the subsequent shape of the vortex was tracked forward in time using the standard fourth-order Runge-Kutta algorithm. The initial vortex was split into a 
number of equal increments in the variable $s$ and the subsequent motion of each point (corresponding to a fixed value of $s$ ) was computed. The gradients of $\vec{x}$ along the vortex were evaluated using central difference formulae and the integrations along the vortex were carried out using Simpson's rule. The vortex core was assumed to be in a state of solid body rotation with no axial flow along the core; it is easily shown (refs. 14 and 15) that $\mu$ in equations (13) and (16) is related to the core radius a by

$$
\mu=e^{-3 / 4} a,
$$

in such circumstances. Other core velocity distributions may be assumed but the effect on the overall vortex motion is weak and only affects the constant of proportionality in equation (20). In all cases, the dimensionless vortex core was assumed small $(a=0.02)$ and was held constant during the integration.

Consider first the case where the vortex develops in an otherwise stagnant flow (with $U_{b}=0$ in equation (11)) above the wall. Computed results are shown in figure 3 for a case with initial amplitude $A=0.5$, angle of inclination $\alpha=45^{\circ}$ and spread parameter $\beta=20$. Initially the self-induced velocities predicted by the Biot-Savart law are quite large near $s=0$ where the vortex curvature is largest; thus it proved necessary to use rather small time steps in order to avoid the occurrence of "wiggles" and sharp corners in the vortex shape. A time step of $\Delta t=0.0002$ was used in the integrations with a uniform mesh size of $\Delta s=0.005$; initially the value of $l$ used was 2 and the computation thus started with 800 points along the vortex. As time increased and the vortex disturbance spread to the side, the value of $l$ was increased to $\ell=3.0$ at $t=0.03$ and then to $\ell=4$ at $t=0.06$. With an increased number of points on the vortex, the calculations become very time consuming and were stopped at $t=0.072$ when the general trends were evident. The vortex evolution in top, side, and end views is displayed in figure 3 where the vortex position has been drawn every 40 time steps. It may be observed that the head of the vortex (corresponding to the tip of the distortion) moves rapidly backward and ultimately moves downward toward the wall. The rate of movement of the vortex head should be contrasted with the relatively slow movement of the straight portions of the vortex which advance upstream in the velocity field of the image. It is evident that the vortex evolves in such a manner so that high local curvature is rapidly diminished. The portion of the curved part of the vortex nearest the wall begins to develop "legs" which curl backward in a counter-clockwise direction; the disturbance moves down toward the wall and spreads in the spanwise direction. With the evolution of the vortex legs, new subsidiary hairpin heads evolve in the spanwise direction to the side of the main disturbance. By $t=0.072$ the general trend is well established; the disturbance is expected to continue to propagate to the side without significant amplification in the streamwise direction, as the vortex turns itself into a "corkscrew" shape. This pattern of evolution appears to be independent of the shape and amplitude of the initial distortion and was also obtained for different initial disturbance configurations (ref. 16). It is important to note that although the calculations were carried out for a stagnant background flow, the same evolution occurs for a background uniform flow with the exception that the changing vortex is progressively convected to the right (for $\left.U_{b}>0\right)$.

When the vortex is convected in a shear flow, the changes in the vortex trajectory are rather more dramatic and the most important effect is 
associated with an increase in extent of the vortex in the streamwise direction. Consider the following simple shear flow

$$
U_{b}(y)= \begin{cases}y V / y_{s}, & y \leq y_{s} \\ v, & y>y_{s},\end{cases}
$$

which is linear near the wall and then is uniform for $y>y_{s}$; the situations of interest correspond to a disturbance developing in a strong shear flow. Vortex trajectories for a number of cases have been obtained in reference 16; here the results of one representative case will be described. For the initial vortex configuration in equation (9), the self-induced velocities near the head of the vortex are initially about 25 units to the left. For the case detailed here, $V=250$ and $y_{s}=1.5$; thus the uniform flow, at distances far from the wall, has a speed about 10 times larger than the maximum self-induced velocities. This simulation therefore describes the evolution of a three-dimensional disturbance riding on a strong shear flow. The computed trajectory is depicted in figure 4 where the vortex position is plotted about 30 time steps $(\Delta t=0.0002)$ and the initial vortex parameters are the same as for the case depicted in figure 3. It may be observed that the streamwise extent of the initial hairpin vortex grows as it convects downstream in the shear flow. The vortex head lifts away from the wall and eventually rises into the uniform flow region. The vortex legs, which develop outboard of the vortex head, begin to move progressively toward the wall and to trail behind the undisturbed two-dimensional portion of the vortex. With the development of the legs, new subsidiary hairpin vortices begin to form and develop to the sides of the original disturbance; as the subsidiary hairpin vortices form, the heads bend back and rise from the wall. The calculation was carried out to $t=0.06$ and during the integration it was necessary to increase $\&$ to 3 and then to 4. A continuation of the integration is depicted in figure 5 up to $t=0.078$ with the vortex position plotted every 15 time steps. It may be observed that the trends seen in figure 4 continue until eventually the vortex legs, immediately to the side of the primary hairpin vortex, touch the wall just after $t=0.078$, thus terminating the computation.

The present results provide a detailed time history of the evolution of a vortex in a shear flow; a major effect of the shear flow is to spread the original distortion dramatically in the streamwise direction. Further calculations (ref. 16) show that increased levels of shear lead to an increased rate of spreading in the streamwise direction. The disturbance also spreads rapidly in the spanwise direction producing a series of similar distortions referred to here as subsidiary vortices. It should be noted that the present results are similar to those obtained by Aref and Flinchem (ref. 19) who used a "localized induction" method due to Hama (ref. 20): in this approach, the image vortex is ignored (as well as the effect of most of the main vortex) and the self-induced velocity is evaluated from the contribution due to a small arc surrounding the point in question. The computed results are also in broad agreement with the experimental observations of Acarlar and Smith (refs. 4 and 5) who artificially created and observed the motion of hairpin vortices in a sub-critical boundary-layer flow; these authors noted: (1) the tendency for the vortex head to bend back and rise from the wall; (2) the trend for the vortex legs to move downward toward the wall and (3) the evolution of subsidiary hairpin vortices outboard of the primary hairpin vortex. 


\section{THE BOUNDARY LAYER}

As the hairpin vortex is convected in the shear flow, it induces a complex unsteady three-dimensional boundary-layer flow on the wall and the nature of this viscous response to the motion is considered in this section. At any instant, the velocity induced by the moving vortex is

$$
\vec{u}\left(\vec{x}_{0}, t\right)=\operatorname{sgn}(\Gamma)\left[\int_{-\infty}^{\infty} \frac{\partial \vec{x}_{h}}{\partial s} \times \frac{\left(\vec{x}_{0}-\vec{x}_{h}\right)}{\left|\vec{x}_{0}-\vec{x}_{h}\right|^{3}} d s-\int_{-\infty}^{\infty} \frac{\partial \vec{x}_{i}}{\partial s} \times \frac{\left(\vec{x}_{0}-\vec{x}_{i}\right)}{\left|\vec{x}_{0}-\vec{x}_{i}\right|^{3}} d s\right],
$$

where $\vec{x}_{0}$ is a vector to any location in the flow which is not on the hairpin vortex; $\vec{x}_{h}$ and $\vec{x}_{i}$ are vectors to the hairpin and image vortices respectively. The inviscid velocity distribution near the wall is obtained by taking the limit as $y \rightarrow 0$ in equation (22) and this results in expressions for the streamwise and spanwise velocity distributions, $U_{W}(x, z, t)$ and $W_{W}(x, y, z)$ respectively. Calculation of the full unsteady three-dimensional boundary-layer development requires computational resources which were beyond what was available in the present study; however in order to gain an appreciation of the nature of the viscous flow near the wall, the evolution of the boundary layer was calculated on the symmetry plane of the hairpin $z=0$ using a procedure outlined in reference 21 . Denoting the limit of $U_{W}$ as $z \rightarrow 0$ by $U_{\infty}(x, t)$, it is easily shown (ref. 16) that

$$
U_{\infty}(x, t)=4 \operatorname{sgn}(\Gamma) \int_{0}^{\infty} \frac{\left(y_{h} \frac{\partial z_{h}}{\partial s}-z_{h} \frac{\partial y_{h}}{\partial s}\right)}{\left[\left(x-x_{h}\right)^{2}+y_{h}^{2}+z_{h}^{2}\right]^{3 / 2}} d s .
$$

Here $\left(x_{h}, y_{h}, z_{h}\right)$ denote locations along the curve defining the vortex, which change with time. The temporal development of $U_{\infty}$ is depicted in figure 6 . At $t=0$, the streamwise velocity distribution near the wall shows a region of strong deceleration and then acceleration. As time increases, the vortex head moves progressively away from the wall and the maximum inviscid speed near the wall diminishes slightly. At a later stage $(t=0.04)$, the hairpin head has moved further from the wall but has also translated further downstream as the vortex is stretched out in the shear flow; the influence of the moving hairpin head may be seen in figure 6 at $t=0.04$ where a distention has developed in the $U_{\infty}$ distribution near $x=11$. With the passage of time, the distention develops into a local minimum which may be observed at $t=0.05$ near $x=14$, at $t=0.06$ near $x=15$ and then near $x=18$ at $t=0.07$. This effect is directly associated with the moving vortex head which is convected downstream most rapidly and which always induces a region of deceleration-acceleration beneath it. As the vortex begins to develop subsidiary hairpin heads, additional local minima are observed; for example, the local minimum near $x=12$ at $t=0.06$ (and near $x=13$ at $t=0.07$ ) is associated with the subsidiary hairpin heads which first form outboard of the primary hairpin vortex. Note that with increasing time, the streamwise velocity near the wall is influenced over increasing streamwise distances. 
The spanwise velocity distribution near the wall vanishes as $z \rightarrow 0$ but

$$
W_{W} \sim z \theta_{\infty}(x, t)=\ldots \text { as } z \rightarrow 0,
$$

where it may be shown (ref. 16) that

$$
\theta_{\infty}(x, t)=-12 \operatorname{sgn}(\Gamma) \int_{0}^{\infty} \frac{\left[y_{h} \frac{\partial x_{h}}{\partial s}+\left(x-x_{h}\right) \frac{\partial y_{h}}{\partial s}\right]}{\left[\left(x-x_{h}\right)^{2}+y_{h}{ }^{2}+z_{h}{ }^{2}\right]^{5 / 2}} z_{h} d s .
$$

It follows that $\theta_{\infty}$ is proportional to the spanwise velocity near the symmetry plane and the temporal development of $\theta_{\infty}$ is shown in figure 7. It may be seen that, as the hairpin vortex is stretched out in the shear flow, there is a spanwise inflow $\left(\theta_{\infty}<0\right)$ which is of increasing magnitude as time passes; this occurs because of the development and movement of the hairpin legs toward the wall. By $t=0.06$, the $\theta_{\infty}$ distribution is developing a complicated form with very strong inflow toward the symmetry plane occuring at the streamwise location where the vortex legs are closest to the wall. It should be noted that the $\theta_{\infty}$ is not entirely negative and changes sign (even at early times) at an $x$ location to the left of the minimum $\theta_{\infty}$. One other feature of interest is that by $t=0.06$, the streamwise range over which significant values of $\theta_{\infty}$ occur has expanded considerably; to some extent, the induced inflows are similar to that which would be generated by a pair of counter-rotating streamwise vortices (except near the vortex legs).

The boundary layer on the symmetry plane $z=0$ develops independently of the rest of the three-dimensional unsteady boundary layer and does give some insight as to the viscous response to the moving hairpin vortex. The Reyncids number for the boundary-layer flow may be defined by

$$
\operatorname{Re}=U_{0} d / v \text {, }
$$

where $U_{0}$ and $d$ are the self-induced velocity and distance from the wall of the two-dimensional portion of the vortex respectively. A scaled normal coordinate and normal velocity in the boundary layer are defined by

$$
y^{\prime}=y \operatorname{Re}^{1 / 2}, \quad v^{\prime}=v \operatorname{Re} e^{1 / 2} .
$$

In the boundary layer, $u$ and $v^{\prime}$ are symmetric about $z$ while $w$ is an odd function of $z$, viz.:

$$
w \sim z \theta(x, y, t) \text { as } z \rightarrow 0 .
$$

Substituting into the three-dimensional boundary-layer equations and taking the limit $z \rightarrow 0$ leads to the following special form of the boundary-layer equations on the symmetry plane:

$$
\frac{\partial u}{\partial t}+u \frac{\partial u}{\partial x}+v^{\prime} \frac{\partial u}{\partial y^{\prime}}=\frac{\partial U_{\infty}}{\partial t}+U_{\infty} \frac{\partial U_{\infty}}{\partial x}+\frac{\partial^{2} u}{\partial y^{\prime 2}},
$$




$$
\begin{gathered}
\frac{\partial \theta}{\partial t}+u \frac{\partial \theta}{\partial x}+v^{\prime} \frac{\partial \theta}{\partial y^{\prime}}+\theta^{2}=\frac{\partial \theta_{\infty}}{\partial t}+u_{\infty} \frac{\partial \theta_{\infty}}{\partial t}+\theta_{\infty}^{2}+\frac{\partial^{2} \theta}{\partial y^{\prime}}, \\
\frac{\partial u}{\partial x}+\frac{\partial v^{\prime}}{\partial y^{\prime}}+\theta=0,
\end{gathered}
$$

with the boundary conditions

$$
\theta=u=v^{\prime}=0 \quad \text { at } \quad y^{\prime}=0 ; \quad u \rightarrow U_{\infty}, \quad \theta \rightarrow \theta_{\infty} \text { as } y^{\prime} \rightarrow \infty \text {. }
$$

To solve this system, it is convenient to introduce functions $\phi\left(x, y^{\prime}, t\right)$ and $\psi\left(x, y^{\prime}, t\right)$ defined by

$$
\begin{gathered}
\theta=\frac{\partial \phi}{\partial y}, \quad \phi(x, 0, t)=0, \\
u=\frac{\partial \psi}{\partial y}, \quad v=-\frac{\partial \psi}{\partial x}-\phi, \quad \psi(x, 0, t)=0,
\end{gathered}
$$

and for which the continuity equation is identically satisfied.

The streamwise coordinate ranges from $-\infty$ to $\infty$ and for the numerical computations, it is convenient to transform to a new variable $\xi$ defined on the finite range $(-1,1)$ by

$$
\xi=\frac{2}{\pi} \tan ^{-1}\left[\frac{x-x_{\ell}(t)+\pi}{\pi}\right] .
$$

Here $x_{0}(t)$ represents the instantaneous streamwise location of the undistu bed two-dimensional portion of the vortex. The factors of $\pi$ were chosen with the objective of transforming the streamwise position of the trailing legs of the vortex to locations near $\xi=0.5$. Generally an objective of a transformation like equation (35) is to expand regions where the streamwise velocity distribution is undergoing intense variations; in the present case, this is a difficult task since the locations of severe variation in $U_{\infty}$ are constantly shifting in time. It was found by trial and error (using many transformations like (35)) that the form selected for the transformation was important and that unless particular care was taken to properly represent regions of intense variation in $\theta_{\infty}$ and $U_{\infty}$, significant inaccuracies occurred in the numerical boundary-layer solutions. The transformation in equation (35) does a reasonable job at accomplishing the aforementioned objectives but becomes increasingly unsatisfactory as the hairpin legs move closer to the wall (at $x$ locations which become progressively less than $x_{\ell}$ with increasing time). It is possible, for problems of this nature where the zones of intense variation in the inviscid distributions constantly shift with time, that a time-dependent series of adaptive transformations might be more satisfactory.

The boundary-layer computations were initiated at $t=0$ when it was assumed the effects of viscosity became abruptly important near the wall. For all $t>0$, a thin unsteady boundary layer develops on the wall and in order to take into account the fact that the boundary layer thickens initially proportional to $t^{1 / 2}$, Rayleigh variables defined by 


$$
u=U_{\infty} \frac{\partial \psi}{\partial \eta}, \quad \theta=\theta_{1} \text { erf } n+\theta_{2} \frac{\partial \Phi}{\partial \eta}, \quad n=\frac{y}{2 \sqrt{t}},
$$

were used. Here the functions $\psi$ and $\Phi$ are defined by

$$
\psi=2 \sqrt{t U_{\infty}} \psi, \phi=2 \sqrt{t}\left[\theta_{1} I(n)+\theta_{2} \Phi\right] \text {, }
$$

where $I(n)$ is the integral of the error function

$$
I(n)=\int_{0}^{n} \operatorname{erf} n d \eta=n \operatorname{erf} n+\frac{1}{\sqrt{\pi}}\left(e^{-n^{2}}-1\right) \text {. }
$$

In this formulation (refs. 15 and 16), it is desirable to decompose $\theta_{\infty}$ into two parts according to $\theta_{\infty}=\theta_{1}+\theta_{2}$, both of which are of one sign for all $x$; the following decomposition is not unique and was obtained by trial and error

$$
\begin{gathered}
\theta_{1}(x, t)=\int_{0}^{\infty} \frac{\left[\left(x-x_{l}\right)^{2}+3\right]^{1 / 2}}{\left[\left(x-x_{h}\right)^{2}+y_{h}^{2}+z_{h}^{2}\right]^{5 / 2}} e^{-s} d s, \\
\theta_{2}(x, t)=\theta_{\infty}(x, t)-\theta_{1}(x, t) .
\end{gathered}
$$

This was found to give satisfactory results.

A set of two coupled equations for $\psi$ and $\Phi$ is readily obtained from equations (29) and (30). A mesh was selected in the $\xi \eta$ plane and the boundary-layer solution was advanced forward from $t=0$ using a Crank-Nicolson type method (refs. 15 and 16). For a given value of $t$, the solution at $\xi= \pm 1$ was advanced on time step and then the solution in the interior $|\xi|<1$ could be computed iteratively. In a typical computation, the outer flow conditions were imposed at $n=6$ as an approximation, the time step was $\Delta t=0.002$ and there were 201 and 121 mesh points in the $\xi$ and $n$ directions respectively.

The complete development of the flow patterns in the symmetry plane is described in reference 16 and here only a brief summary is given. Shortly after the initiation of the boundary-layer motion, separation was observed to occur in the form of regions of recirculation. Unlike two-dimensional separation in which the streamlines form closed loops, the three-dimensional separations observed here involve a spiral motion toward a focus as depicted in figure 8, where the instantaneous streamlines at $t=0.05$ are plotted. The point labelled $F 1$ is a focus at which the instantaneous flow is leaving the symmetry plane; streamlines near Fl all spiral in toward the focus. The stagnation point $s i$ is a saddlepoint of detachment and along the limiting streamline emanating from $S 1$ the flow is away from the wall and toward the focus at Fl. As useful points of reference, the instantaneous streamwise 
locations of the hairpin vortex head and the two-dimensional portion of the vortex are indicated at the top of figure 8 by the square and triangular symbols respectively. It may be observed that the significant developments in the boundary layer are occuring behind the two-dimensional part of the vortex. At a later stage, as the hairpin legs approach the wall, multiple stagnation points appear in the flow on the wall (ref. 16) and the flow patterns become rather complex. However the important point is that the latter stages of the integration are characterized by rapid boundary-layer growth on the symmetry plane in the region behind the two-dimensional part of the vortex; the growth occurs in several locations corresponding to those near the current streamwise positions of the vortex legs as well as behind the heads of the subsidiary hairpin vortices.

\section{DISCUSSION}

In this study, some aspects of the dynamics of hairpin vortices in a shear flow near a wall have been examined on a theoretical basis. The results of the present investigation support and complement the findings of the flow visualization studies of Acarlar and Smith (refs. 4 and 5). In particular, specific conclusions are: (1) Hairpin vortices in a shear flow develop subsidiary hairpin vortices which spread and multiply in the spanwise direction and (2) convected hairpin vortices induce a pressure distribution near the wall which ultimately leads to strong boundary-layer growth in the region behind the vortex. In relation to this last point, it is reasonable to expect that, as the rapidly thickening boundary layer penetrates out into the inviscid region, a roll-over into a new secondary hairpin vortex will occur. Such an event constitutes a strong unsteady viscous-inviscid interaction. Although it has not been possible to compute the evolution of the interaction using the methods described in this paper, the experimental results of Acarlar and Smith (refs. 4 and 5 ) confirm that secondary hairpin vortices do evolve in the manner described here. This process is clearly regenerative and shows how one hairpin vortex can lead to the creation of another through an interaction with the viscous flow near the wall. Finally it is worthwile to note that the boundary-layer evolution has been computed only on the symmetry plane in this study. It is entirely possible that the interaction between the boundary layer and the outer inviscid flow may initiate at an earlier stage at locations off the symmetry plane. Previous studies of vortex motions in two dimensions (refs. 6, 7, and 15) indicate that a vortex will evoke an eruptive response from the viscous flow near the wall more rapidly if the vortex is stronger and/or is moved closer to the wall. In the present case, the closest approach to the wall is made by the hairpin vortex legs at locations off the symmetry plane; it is in this region where the most violent boundary-layer response might be anticipated.

\section{ACKNOWLEDGEMENTS}

The authors would like to thank the Air force Office of Scientific Research for support of this work under contract number F49620-85-C-0033; some computations were performed at the Pittsburgh Supercomputing Center. Some of the calculations were carried out at ICOMP at NASA Lewis Research Center whose support is appreciated. 


\section{REFERENCES}

1. Theodorsen, T.: Mechanisms of Turbulence. Proceedings of the Second Midwestern Conference on Fluid Mechanics, Ohio State University, 1952, pp. 1-18.

2. Head, M.R.; and Bandyopadhyay, P.: New Aspects of Turbulent BoundaryLayer Structure. J. Fluid Mech., vol. 107, June 1981, pp. 297-337.

3. Wallace, J.M.: On the Structure of Bounded Turbulent Shear-flow: A Personal View. Developments in Theoretical and Applied Mechanics, vol. 11, G.R. Karr et al., eds., Univ. of Alabama, Huntsville, AL, 1982, pp. 509-522.

4. Acarlar, M.S.; and Smith, C.R.: A Study of Hairpin Vortices in a Laminar Boundary Layer. Part I. Hairpin Vortices Generated by a Hemisphere Protuberance. J. Fluid Mech., vol. 175, Feb. 1987, pp. 1-41.

5. Acarlar, M.S.; and Smith, C.R.: A Study of Hairpin Vortices in a Laminar Boundary Layer. Part II. Hairpin Vortices Generated by Fluid Injection. J. Fluid Mech., vol. 175, Feb. 1987, pp. 43-83.

6. Doligalski, T.L.; and Walker, J.D.A.: The Boundary Layer Induced by a Convected Two-Dimensional Vortex. J. Fluid Mech., vol. 139, Feb. 1984, pp. 1-28.

7. Walker, J.D.A.; Smith, C.R.; Cerra, A.W.; and Doligalski, T.L.: The Impact of a Vortex Ring on a Wall. J. Fluid Mech., vol. 181, Aug. 1987, pp. $99-140$.

8. Elliott, J.W.; Cowley, S.J.; and Smith, F.T.: Breakdown of Boundary Layers: (I) On Moving Surfaces; (II) In Semi-Similar Unsteady Flow; (III) In Fully Unsteady Flow. Geophys. Astrophys. Fluid Dyn., vol. 25, no. 1-2, Aug. 1983, pp. 77-138.

9. Peridier, V.J.; Smith, F.T.; and Walker, J.D.A.: Methods for the Calculation of Unsteady Separation. AIAA Paper 88-0604, Jan. 1988.

10. Moin, P.; and Kim, J.: Numerical Investigation of Turbulent Channel Flow. J. Fluid Mech., vol. 118, May 1982, pp. 341-377.

11. Moin, P.: Analysis of Turbulent Data Generated by Direct Numerical Simulations. AIAA Paper 87-0194, Jan. 1987.

12. Callegari, A.J.; and Ting, L.: Motion of a Curved Vortex Filament With Decaying Vortical Core and Axial Velocity. SIAM J. Appl. Math., vol. 35, no. $1,1978, \mathrm{pp} .148-174$.

13. Moore, D.W.: Finite Amplitude Waves of Aircraft Trailing Vortices. Aeronaut. Q., vol. 23, pt. 4, Nov. 1972, pp. 307-314.

14. Moore, D.W.; and Saffman, P.G.: The Motion of a Vortex Filament With Axial Flow. Philos. Trans. R. Soc. London Ser. A, vol. 272, 1972, pp. 403-429. 
15. Ersoy, S.; and Walker, J.D.A.: The Viscous Flow Induced Near a Wall by Counter-Rotating Vortex Pairs and Vortex Loops. Report FM-8, Dept. of Mechanical Engineering and Mechanics. Lehigh University, 1985. (Also AFOSR-87-0382TR, Avail. NTIS, AD-A179462.)

16. Hon, T.-L.; and Walker, J.D.A.: An Analysis of the Motion and Effects of Hairpin Vortices. Report FM-11, Dept. of Mechanical Engineering and Mechanics, Lehigh University, 1987. (Also, AFOSR-87-13892TR, Avail. NTIS, AD-A187261).

17. Dhanak, M.R.; and DeBernardinis, B.: The Evolution of an Elliptic Vortex Ring. J. Fluid. Mech., vol. 109, Aug. 1981, pp. 189-216.

18. Dhanak, M.R.: Interaction Between a Vortex Filament and an Approaching Rigid Sphere. J. Fluid Mech., vol. 110, Sept. 1981, pp. 129-148.

19. Aref, H.; and Flinchem, E.P.: Dynamics of a Vortex Filament in Shear Flow. J. Fluid Mech., vol. 148, Nov. 1984, pp. 477-497.

20. Hama, F.R.: Progressive Deformation of a Curved Vortex Filament by Its Own Induction. Phys. Fluids, vol. 5, no. 10, Oct. 1962, pp. 1156-1162.

21. Ersoy, S.; and Walker, J.D.A.: The Boundary Layer Due to a ThreeDimensional Vortex Loop. J. Fluid Mech., vol. 185, Dec. 1987, pp. 569-598.

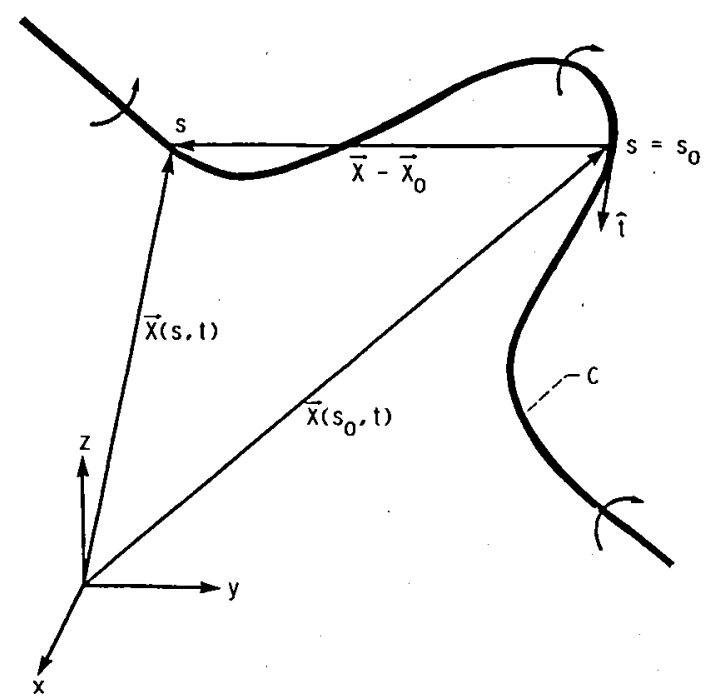

FIGURE 1. - A PORTION OF A VORTEX FILAMENT (SHOWN FOR $\Gamma<0$ ). 


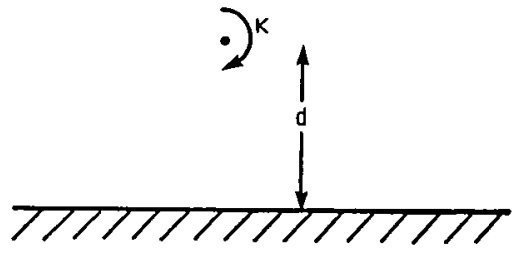

(a) TWO-DIMENSIONAL VORTEX (SIDE VIEW).

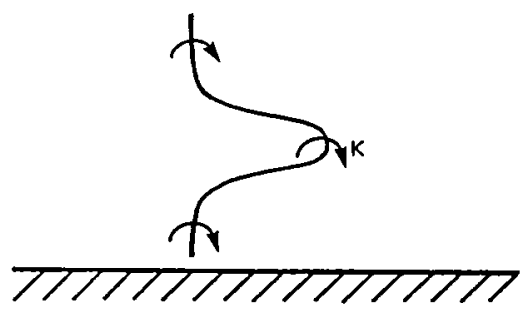

(b) HAIRPIN VORTEX (OBLIQUE VIEW).

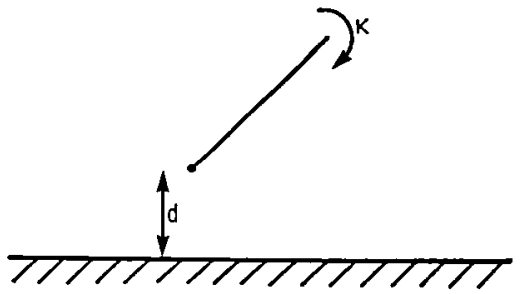

(c) HAIRPIN VORTEX (SIDE VIEW).

FIGURE 2, - VORTEX MOTION ABOVE A WALL.

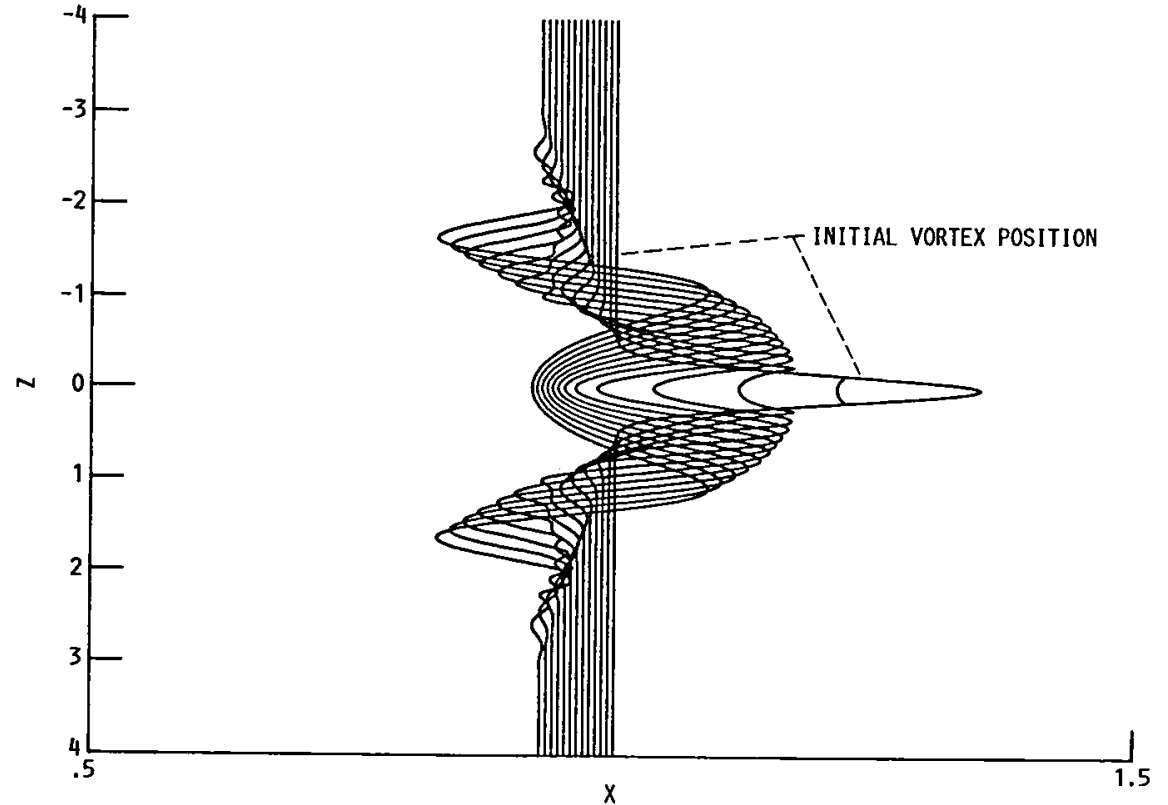

(a) TOP VIEW.

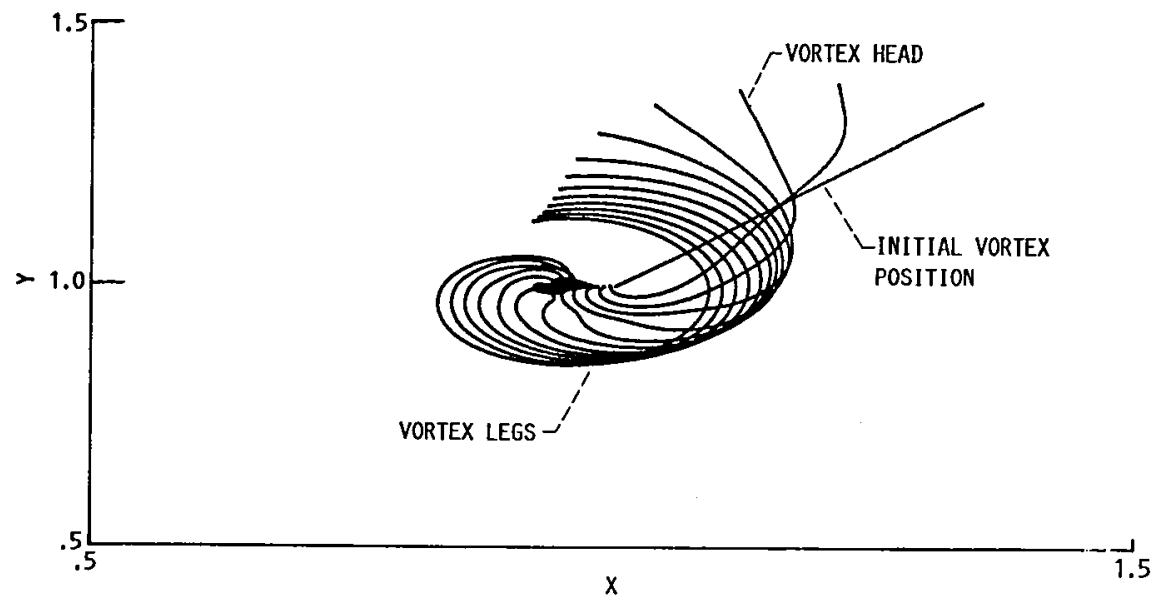

(b) SIDE VIEW.

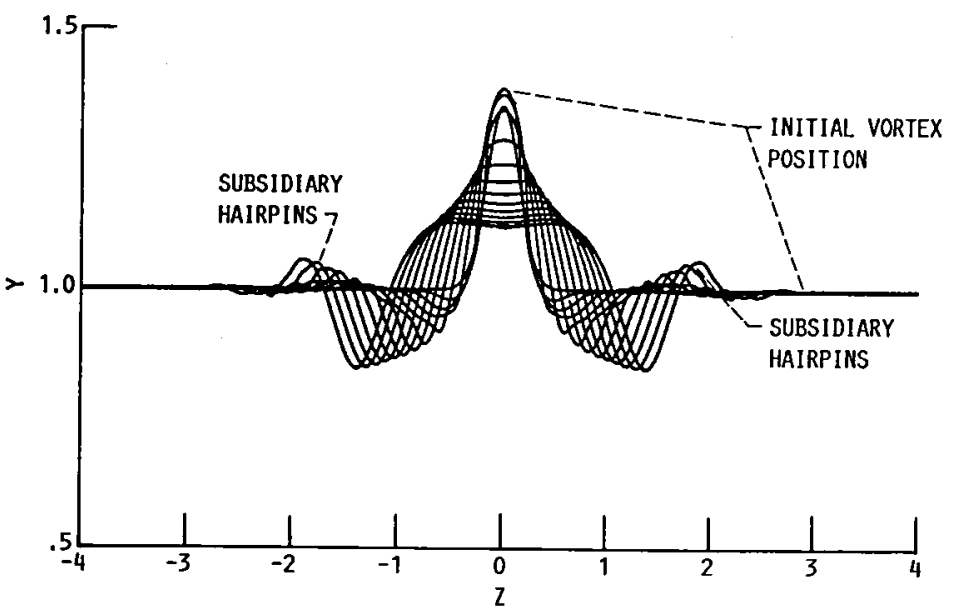

(c) END VIEW.

FiguRE 3. - TEMPORAL DEVELOPMENT FOR A HAIRPIN VORTEX IN A STAGNANT FLOW. 


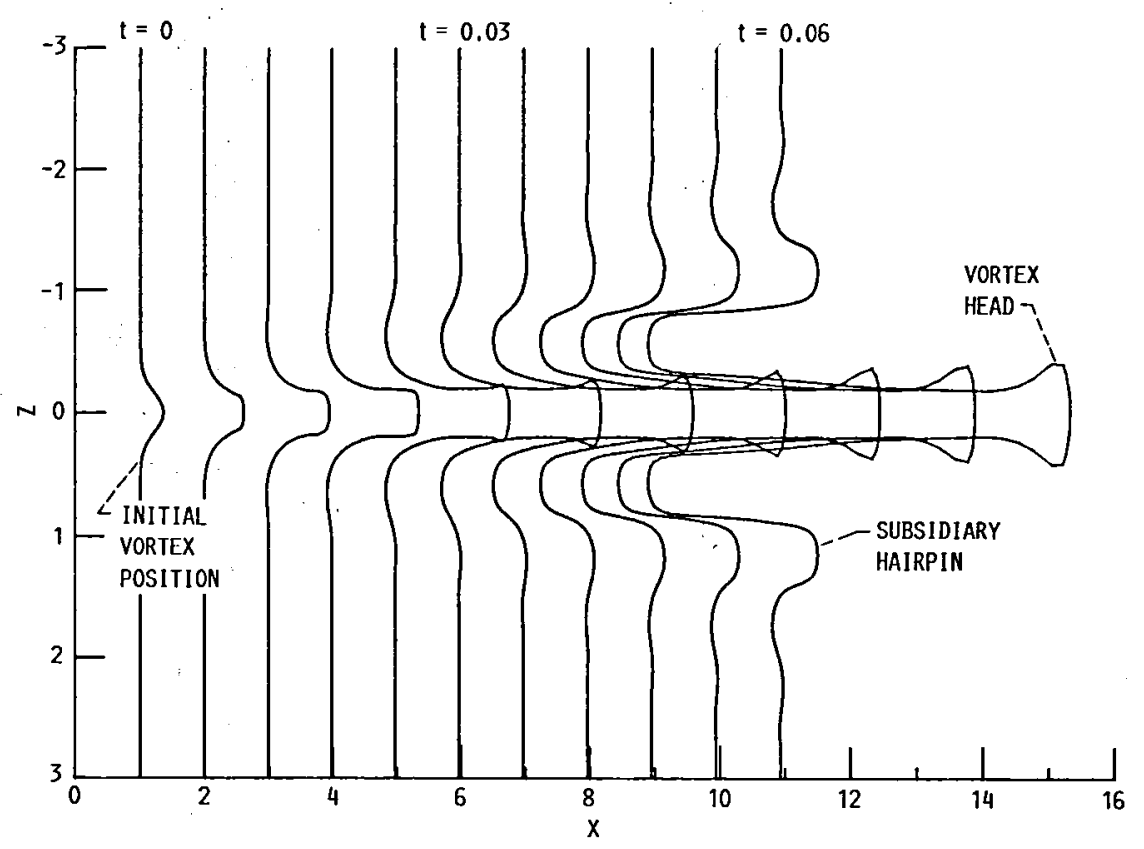

(a) TOP VIEW.

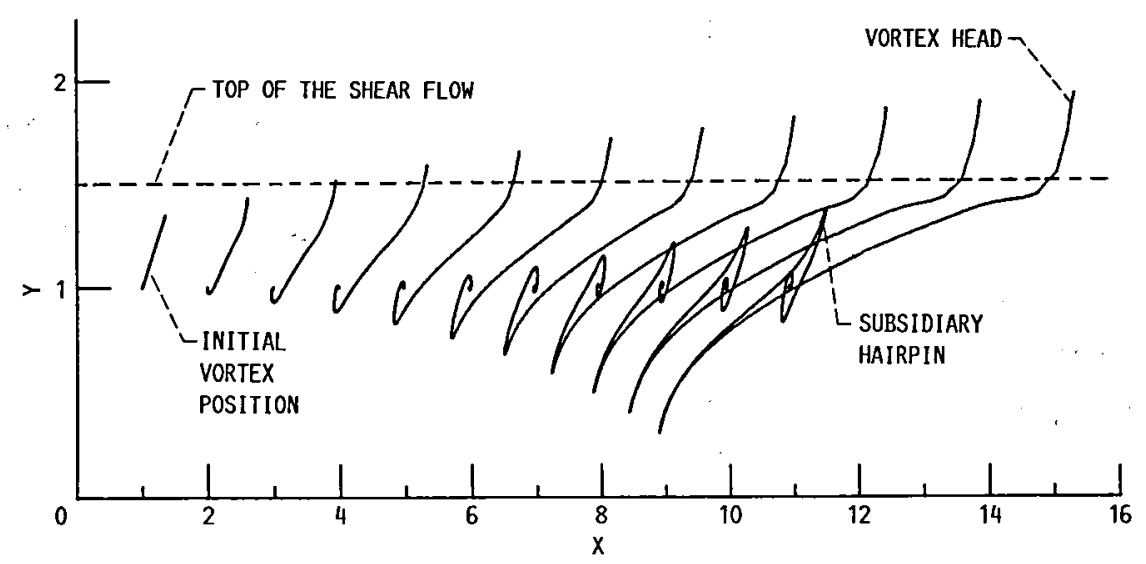

(b) SIDE VIEW.

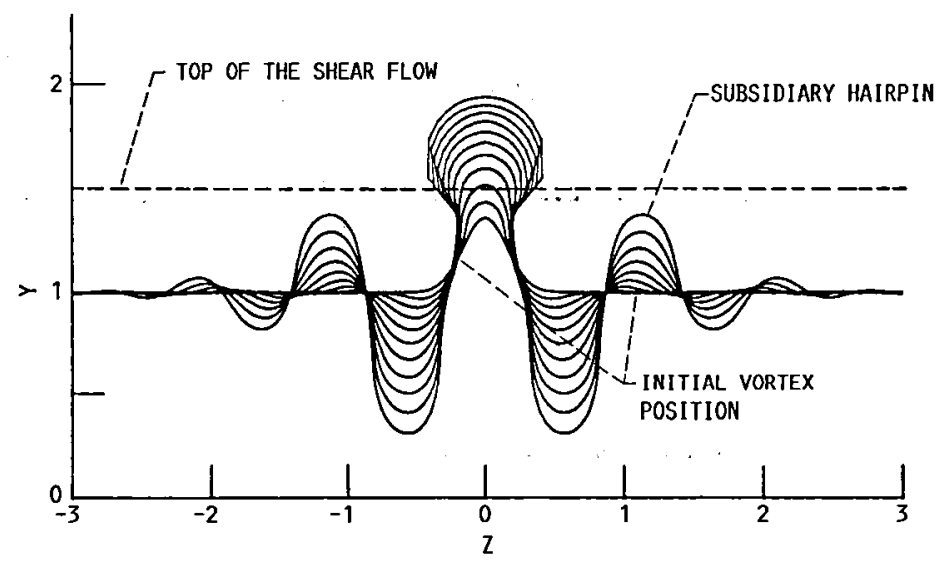

(c) END VIEW.

FIGURE 4. - TEMPORAL DEVELOPMENT FOR A HAIRPIN VORTEX IN A SHEAR FLOW; THE VORTEX POSITION IS PLOTTED EVERY 30 TIME STEPS $(\Delta t=0.0002)$. 


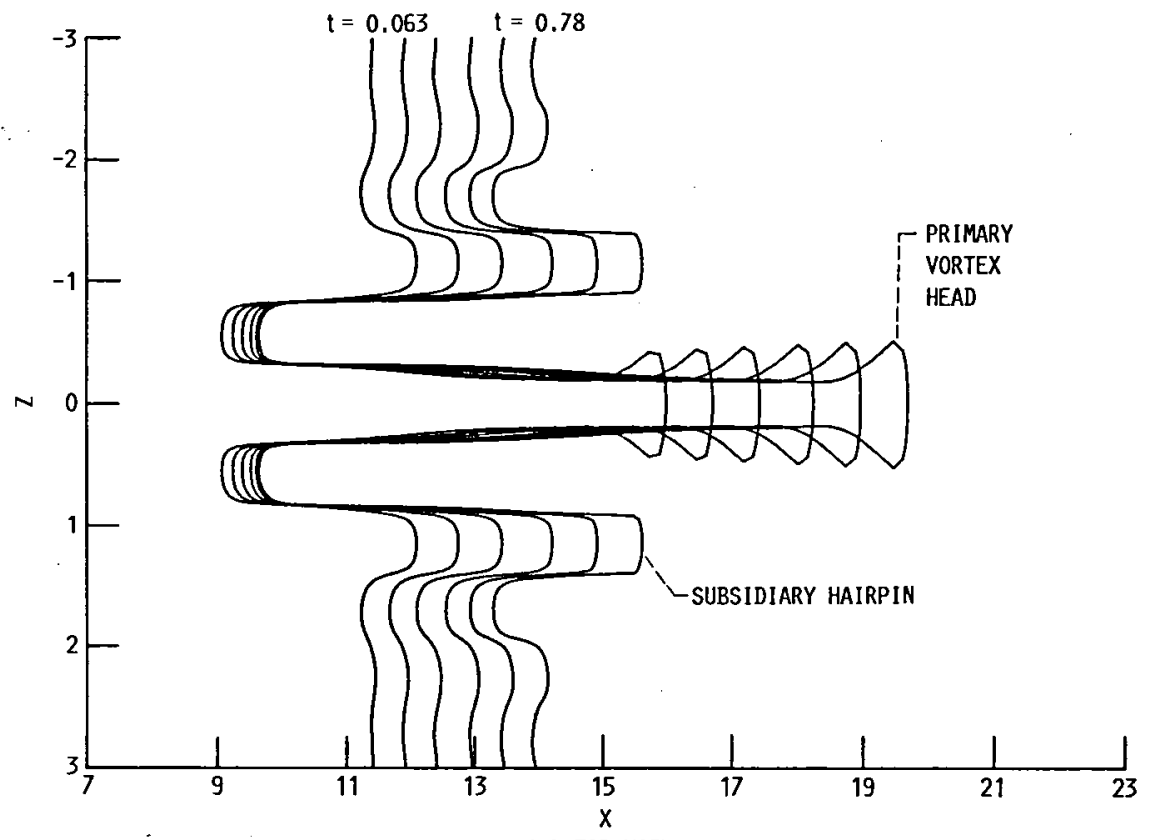

(a) TOP VIEW.

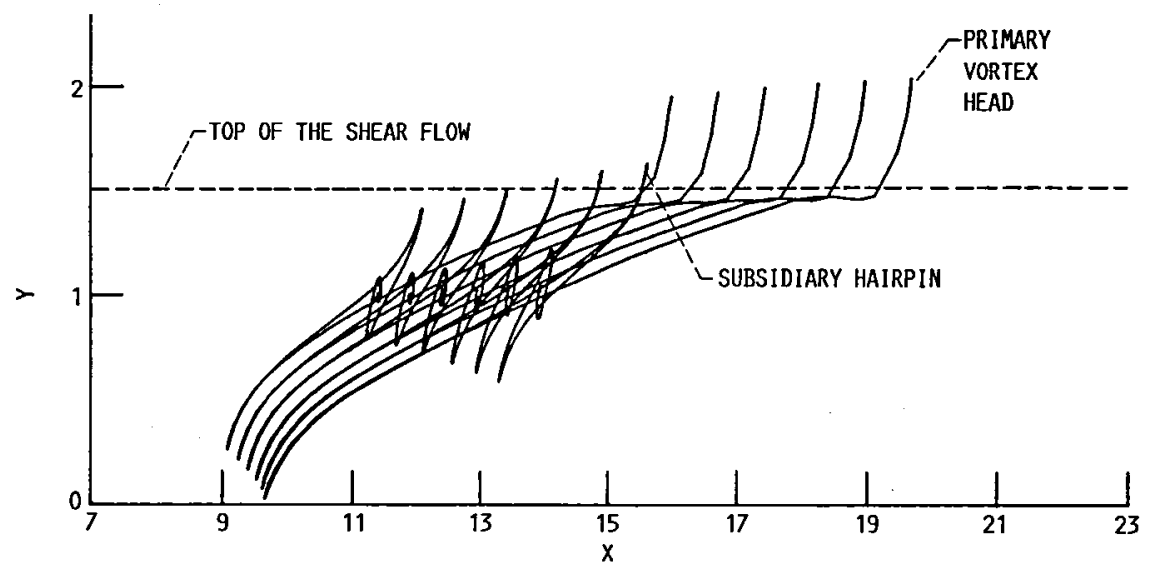

(b) SIDE VIEW.

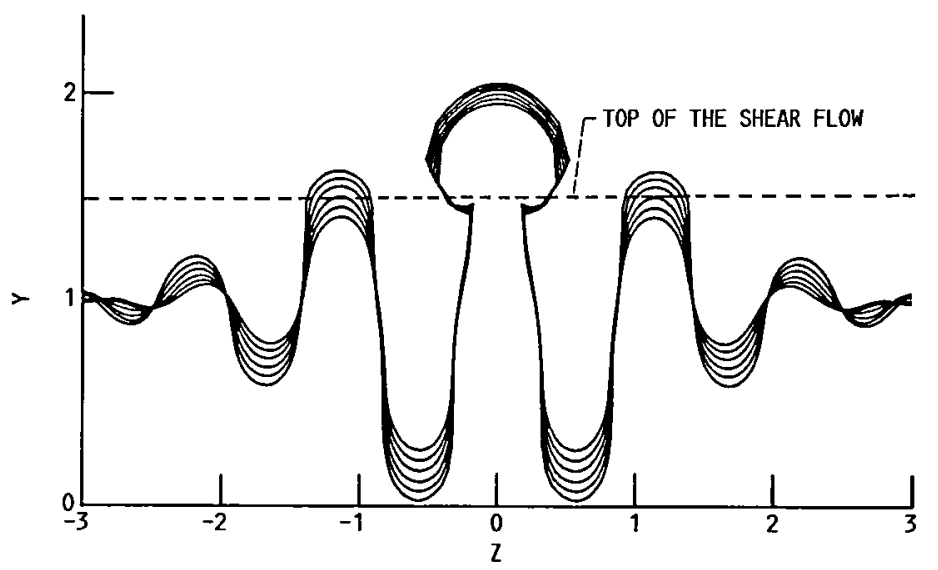

(c) END VIEW.

FIGURE 5. - CONTINUATION OF INTEGRATION OF FIGURE 4: THE VORTEX POSITION IS PLOTTED EVERY 15 TIME STEPS $(\Delta t=0.0002)$. 


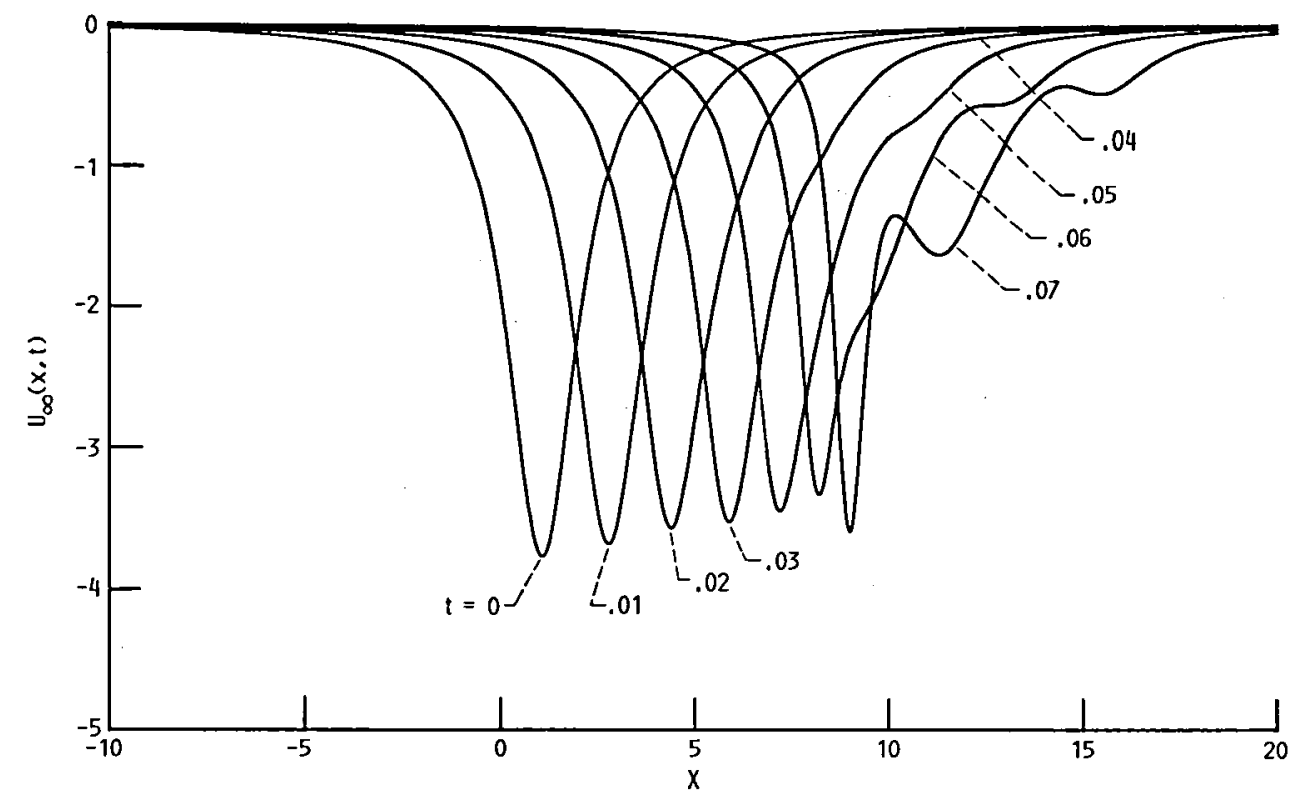

FIGURE 6. - INVISCID STREAMWISE VELOCITY ON THE SYMMETRY PLANE NEAR THE WALL INDUCED BY A MOVING HAIRPIN VORTEX IN SHEAR.

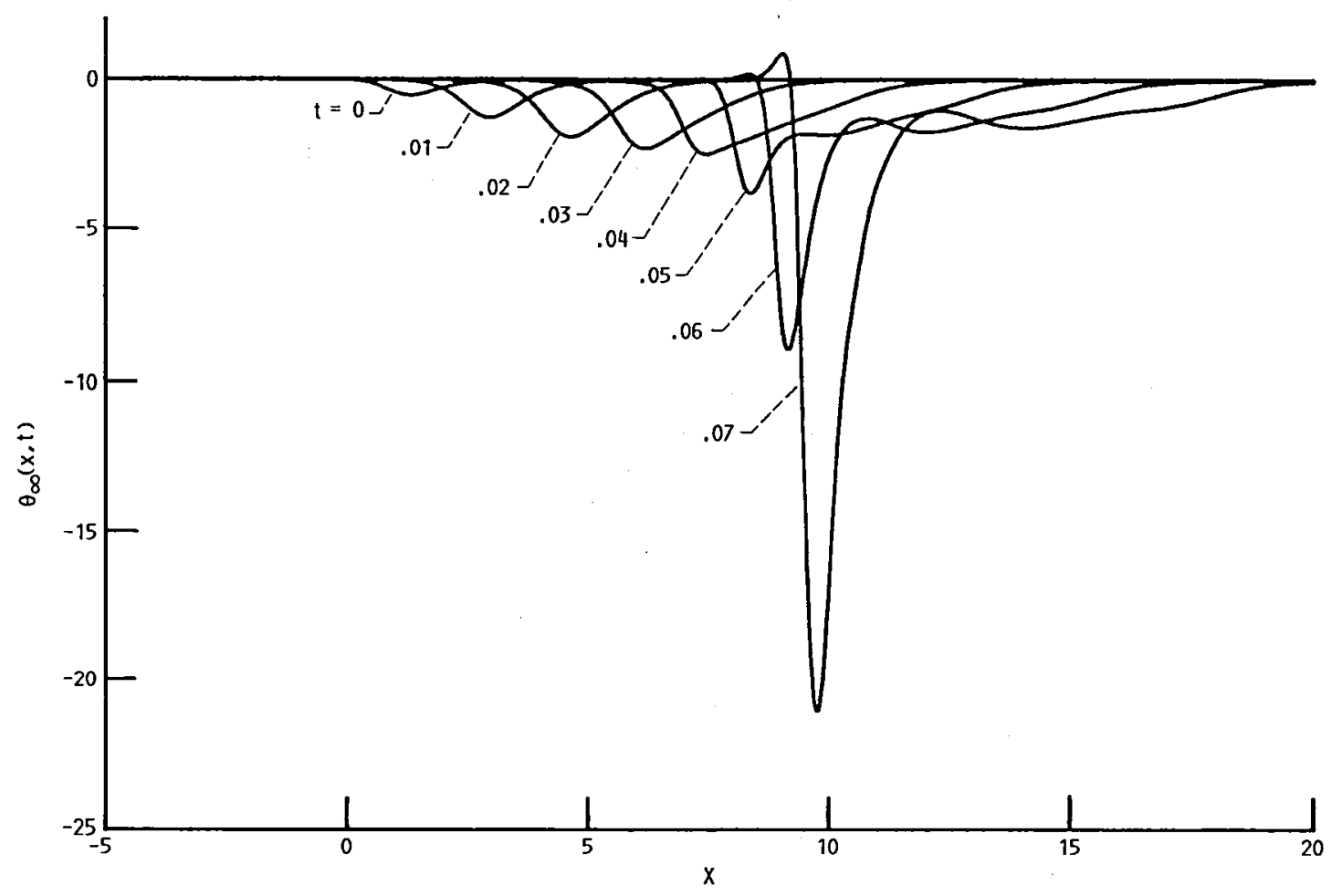

FIGURE 7. - TEMPORAL DEVELOPMENT OF THE SPANWISE VELOCITY DISTRIBUTION NEAR THE SYMMETRY PLANE INDUCED BY THE MOVING HAIRPIN VORTEX IN SHEAR. 


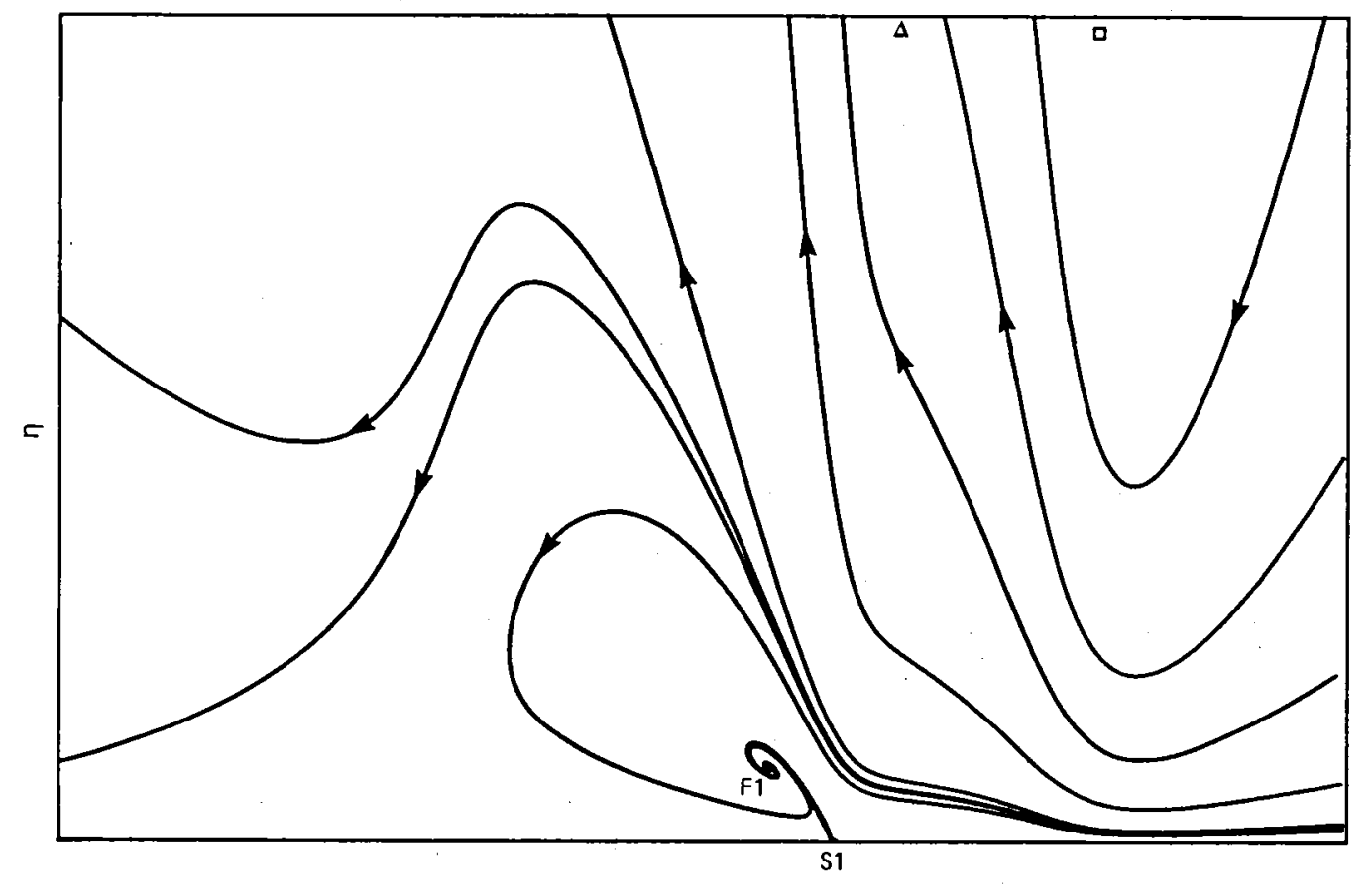

FIGURE 8. - STREAMLINES IN THE SYMMETRY PLANE AT $t=0.05$. 


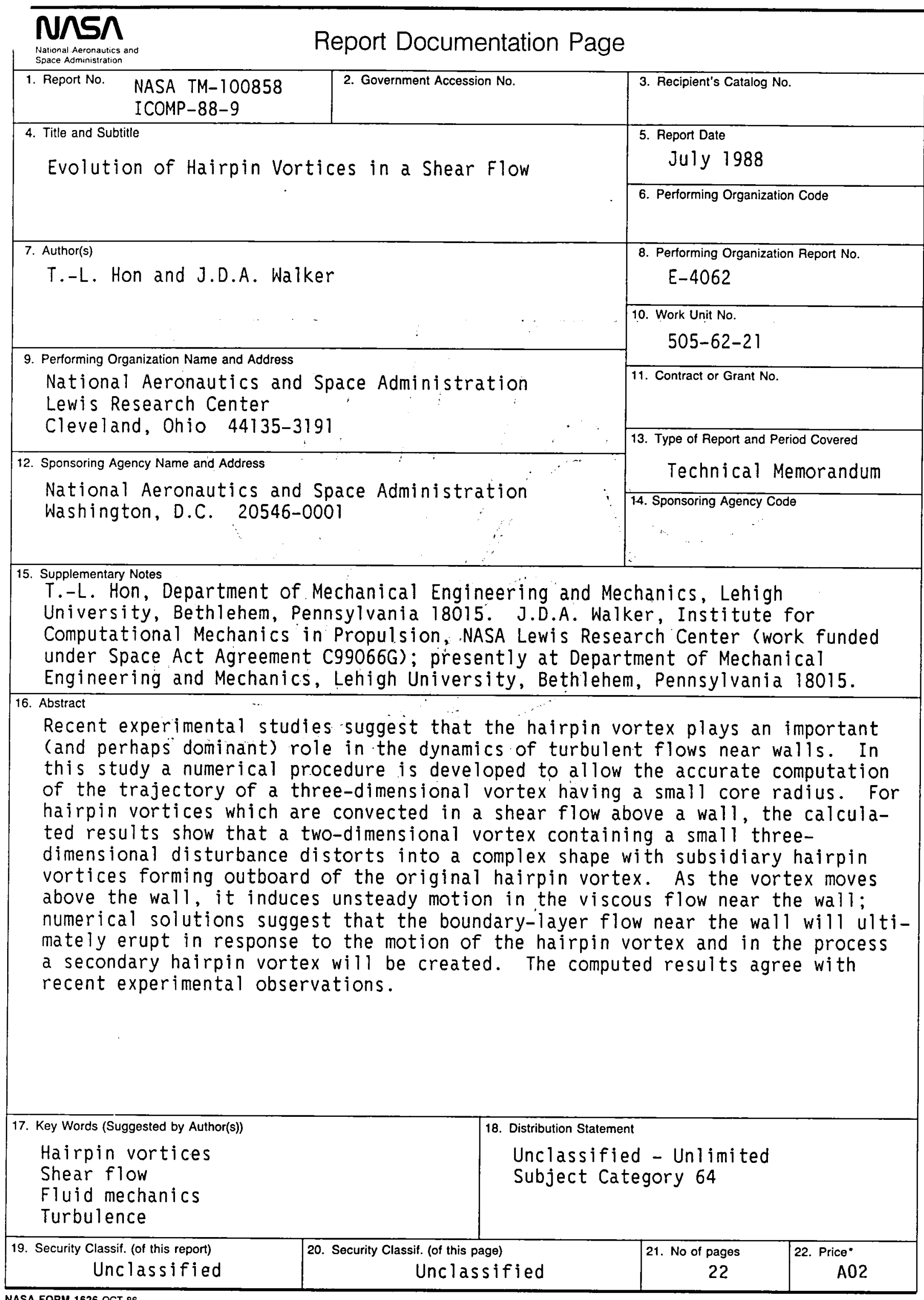


ational Aeronautics and space Administration

Lewis Research Center ICOMP (M.S. 5-3)

Cleveland, Ohio 44135

Otficlal Business

Penalty for Private Use $\mathbf{5 3 0 0}$
FOURTH CLASS MAIL

ADDRESS CORRECTION REQUESTED

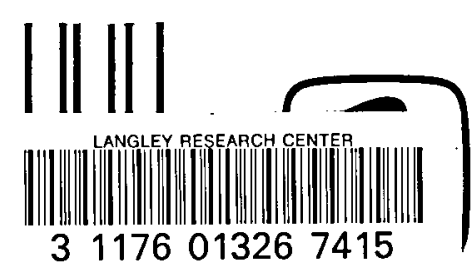

Postage and Fees Paid National Aeronautıcs and Space Admınıstration NASA.451

\section{DO NOT REMOVE SLIP FROM MATERIAL}

R2Y12

Delete your name from this slip when returning material to the library.

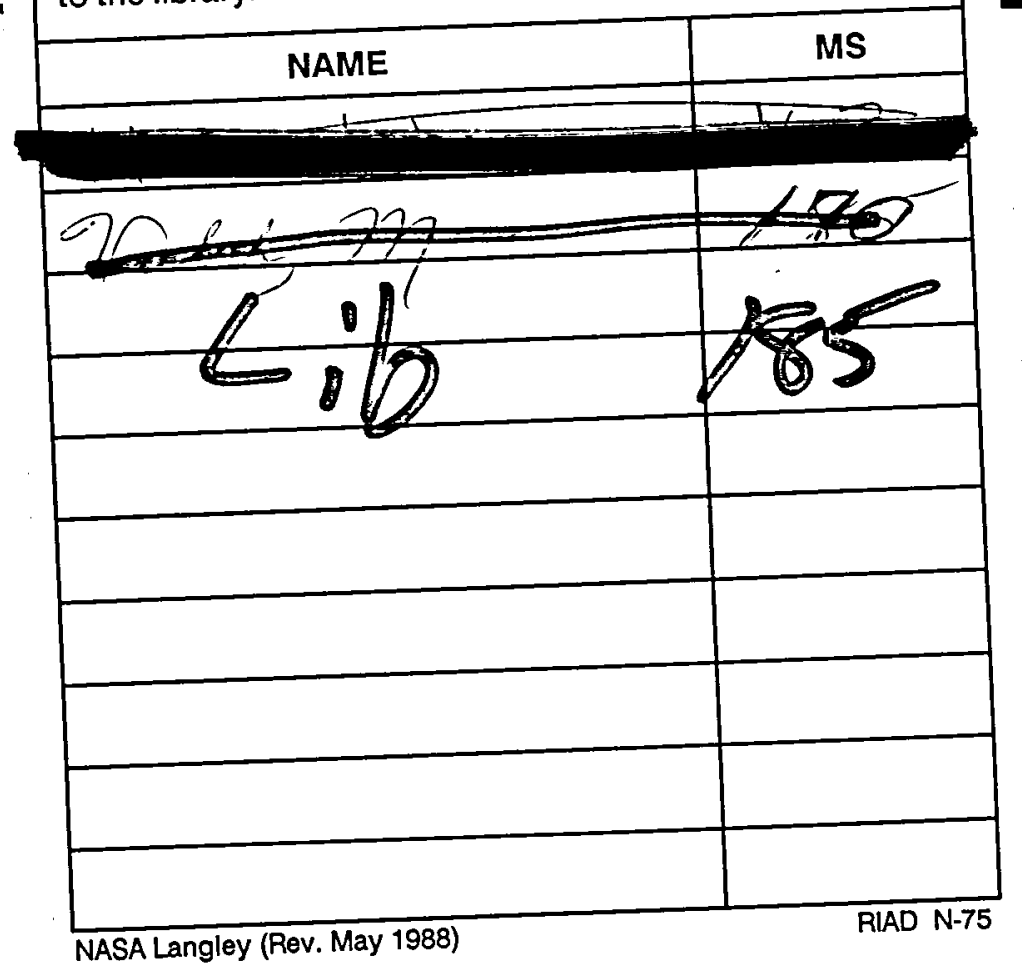

